January 2000

\title{
Acute adenolymphangitis due to bancroftian filariasis in Rufiji district, south east Tanzania
}

D.B. Gasarasi

Muhimbili University College of Health Sciences

Zul Premji

Aga Khan University, zul.premji@aku.edu

P.G.M. Mujinja

Muhimbili University College of Health Sciences

R. Mpembeni

Muhimbili University College of Health Sciences

Follow this and additional works at: http://ecommons.aku.edu/eastafrica_fhs_mc_pathol

Part of the Pathology Commons

\section{Recommended Citation}

Gasarasi, D., Premji, Z., Mujinja, P., Mpembeni, R. (2000). Acute adenolymphangitis due to bancroftian filariasis in Rufiji district, south east Tanzania. Acta Tropica, 75(1), 19-28.

Available at: http://ecommons.aku.edu/eastafrica_fhs_mc_pathol/88 


\title{
Acute adenolymphangitis due to bancroftian filariasis in Rufiji district, south east Tanzania
}

\author{
D.B. Gasarasi a,*, Z.G. Premji a, P.G.M. Mujinja ${ }^{\text {b }}$, R. Mpembeni ${ }^{\text {c }}$ \\ a Department of Parasitology and Medical Entomology, Muhimbili University College of Health Sciences, Box 65011, \\ Dar-es-Salaam, Tanzania \\ ${ }^{\mathrm{b}}$ Department of Behavioural Sciences, Muhimbili University College of Health Sciences, Box 65015, Dar-es-Salaam, Tanzania \\ ${ }^{\mathrm{c}}$ Department of Epidemiology and Biostatistics, Muhimbili University College of Health Sciences, Box 65015,
} Dar-es-Salaam, Tanzania

Received 24 November 1997; received in revised form 11 October 1999; accepted 18 October 1999

\begin{abstract}
A longitudinal prospective surveillance for acute adenolymphagitis (ADL) was carried out in three villages in Rufiji district. A sample population of 3000 individuals aged 10 years and above was monitored fortnightly for a period of 12 months. The annual incidence of ADL was found to be 33 per 1000 population and was significantly higher in males than females (52.7/1000 and 18.7/1000 respectively). ADL episodes were more frequent in the age group of 40 years and above. Individuals with chronic manifestations seemed to be more vulnerable to ADL attacks with $62.2 \%$ of the total episodes occurring in this group. Furthermore, individuals with lymphoedema experienced more frequent acute episodes compared to those with hydrocele and 'normal exposed'. ADL episodes ranged from one to five per annum and the majority of the affected (60.4\%) experienced a single episode. The average duration of an ADL episode was 8.6 days and in $72.5 \%$ of the episodes the affected individuals were incapacitated and unable to do their normal activities for an average duration of 3.7 days. The physical incapacitation associated with ADL episodes emphasizes the significance of lymphatic filariasis as a major public health problem of substantial socio-economic consequences. (C) 2000 Elsevier Science B.V. All rights reserved.
\end{abstract}

Keywords: Filariasis; Adenolymphangitis; Lymphoedema; Tanzania

\section{Introduction}

Lymphatic filariasis due to Wuchereria bancrofti is a disease of major public health significance, affecting millions of people in the tropical areas of Africa, India, South and Central America,

\footnotetext{
* Corresponding author. Tel.: + 255-51-150302/6 ext. 231.

E-mail address: dgasarasi@muchs.ac.tz. (D.B. Gasarasi)
}

(WHO, 1995; Michael et al. 1996). The overt clinical manifestations of hydrocele, elephantiasis and the syndrome of acute filarial fevers, cause a lot of morbidity and stigma associated with the grotesquely enlarged limbs and genitalia (Partono, 1984; WHO, 1992; Evans et al., 1993). However, because the disease is insidious in nature and does not cause any dramatic mortality, it has not received the attention it merits. 
The existing information on the development of lymphatic pathology suggest that the acute filarial fevers, which are often associated with an inflammatory reaction, initiate progressive lymphatic damage, leading to fibrosis and obstruction of the lymph vessels. However, the aetiology of acute adenolymphangitis (ADL) is yet to be well understood and the available information point to bacterial infections, immune responses to parasite antigen and repeated infections with filarial infective larvae (L3) as possible aetiological agents (Evert et al., 1980; Olszewski et al., 1993, 1997; Addis et al., 1994; Shenoy et al. 1998).

Although the chronic manifestations of hydrocele and elephantiasis are associated with reduced agility and impede economic performance, the use of these manifestations alone in the estimation of the global burden of disease due to lymphatic filariasis (World Bank, 1993; Michael et al. 1996) is an underestimate of the level of impact associated with this condition and may have contributed to the low priority accorded to lymphatic filariasis. Reports suggest that the clinical manifestation of adenolymphangitis may be an important cause of loss of work due to lymphatic filariasis (Jordan, 1955; Raghavan, 1969; Evans et al., 1993).

The available information on the impact of ADL is limited and there is paucity of systematic studies characterizing this syndrome. Very little is known concerning the incidence in various endemic areas and there is insufficient information on the level of incapacitation associated with ADL attacks. This information is required from various endemic areas to facilitate a more accurate estimate of the burden of disease due to lymphatic filariasis.

Recently however, systematic studies were initiated by WHO/TDR and carried out as part of a multicentre study, designed to assess the social and economic impact of lymphatic filariasis in six endemic areas (Ghana, Madras, Philippines, Pondicherry, Tanzania and Vellore).

This paper highlights the findings for the Tanzanian study centre, which characterize the ADL syndrome with respect to its incidence, duration and severity.

\section{Materials and methods}

\subsection{Study site}

The study was conducted in Rufiji district, which is located in South East Tanzania. It is one of the four administrative districts of the coast region, with an area of $18000 \mathrm{~km}^{2}$ and a population of 151325 people (71 613 males and 80703 females) according to the 1988 census. Climatically the temperature ranges between 18 and $40^{\circ} \mathrm{C}$. Rainfall is seasonal, the wet season being November to May. The district is bisected by the large Rufiji river which empties into the Indian ocean on the eastern side of the district. The major health problems, as reported in the health centres and dispensaries include: malaria, skin diseases, upper respiratory infections and eye infections. Filariasis is not routinely reported in the health centers and dispensaries.

However, the area is endemic for filariasis, with reported microfilaria rate of $18 \%$, scrotal elephantiasis of $9 \%$, hydrocele rate of $12 \%$ and limb elephantiasis of $0.4 \%$ in the examined population (Matola, 1985). The major economic activities are subsistence farming and fishing.

\subsection{Sampling frame}

Administratively Rufiji district constitute six divisions, 19 wards and 83 registered villages, with approximately 30272 households.

For the purposes of the study, three divisions were purposely selected to represent the ecological differences and variation in economic activities in the district. A division consititutes about nine to twelve villages. Stratified random sampling was used to select the study villages viz, Ikwiriri, Mlanzi and Utete.

\subsection{Study sample}

A house to house cross-sectional morbidity survey was undertaken to delineate the extent of filariasis and other chronic conditions in each of the study villages. During the cross-sectional morbidity survey, individuals aged 10 years and above were included in the survey. The ages below 10 
years were excluded based on the understanding that this age group frequently suffer from bacterial and other viral infections which would have been confounding for the ADL diagnostic criteria.

From the population examined in each of the three villages (Table 1) a random sample of 1000 individuals was selected to total 3000 individuals included in the surveillance for ADL episodes. The sample size of 3000 was an estimate based on the expectation that the incidence of ADL attacks in the endemic population is $2 \%$.

\subsection{Study procedure}

\subsubsection{Surveillance for $A D L$}

In each study village a population of 1000 individuals was followed by three trained research assistants, under the supervision of a clinical officer (these are experienced clinicians managing the health problems at district level) for a period of 12 months. Every 2 weeks on the scheduled dates the research assistant visited the households of the selected individuals. If the individual was present the research assistant completed a screening form with a symptom checklist and returned the screening forms to the clinical officer at the end of the day. If an individual was absent an appointment was made for the next day and if absent on that day, the individual was recorded as absent.

\subsubsection{Criteria for ADL confirmation}

According to the WHO definition (WHO, 1993) an ADL episode would be confirmed if the affected individual presented with localised pain, lymphadenitis and/or/lymphangitis and/or cellulitis and local warmth, with or without systemic manifestations of fever, nausea and vomiting lasting for at least 3 days.

\subsubsection{Confirmation of $A D L$}

The confirmation of ADL was done by the clinical officer in each village, who assessed the screening forms submitted by the research assistants on the day of the visit. Individuals who reported symptoms of fever, local swelling, local pain, lymphadenitis and local warmth were visited within $24 \mathrm{~h}$ of receiving the report.

The clinical officer reconfirmed the symptoms reported by the research assistant on the confirmatory forms. The affected individuals were physically examined to elicit, lymphoedema and/or local swelling, local tenderness and inflammation of the lymphnodes and lymph vessels. In acute filarial lymphangitis the affected lymph vessels are usually inflamed, dilated, varicose and cord like. They are easily palpable and acutely tender. The underlying skin is turgid or oedematous and there are streaks of erythma running along the course of the vessels.

An ADL case was therefore clinically confirmed if the criteria for diagnosis were met, following history taking and physical examination by the clinical officer. A suspected ADL case was confirmed if two of the three major criteria as defined above were met with or without systemic manifestations of fever, nausea and vomiting lasting for at least 3 days.

Active ADL episodes and those which occurred within a 2 week recall period were recorded.

Table 1

Distribution of chronic filariasis, incidence of ADL and microfilaraemia

\begin{tabular}{|c|c|c|c|c|}
\hline \multirow[t]{2}{*}{ Pathology group } & \multicolumn{4}{|l|}{ Village } \\
\hline & $\begin{array}{l}\text { Ikwiriri }(n= \\
1873)\end{array}$ & $\begin{array}{l}\text { Mlanzi }(n= \\
1529)\end{array}$ & Utete $(n=1174)$ & Total $(n=4576)$ \\
\hline ADL (based on recall) per 1000 population & 41 & 46 & 62 & 48 \\
\hline Chronic filariasis & $1.5 \%(29)$ & $4.8 \%(74)$ & $3.2 \%(38)$ & $3.1 \%(141)$ \\
\hline $\begin{array}{l}\text { Annual incidence of ADL (based on surveillance) } \\
\text { per } 1000 \text { population }\end{array}$ & 15 & 53 & 28 & 33 \\
\hline Microfilaria prevalence & $12.8 \%(n=658)$ & $6.1 \%(n=442)$ & $14.4 \%(n=604)$ & $11.6 \%$ \\
\hline
\end{tabular}


Those with active attacks were treated with analgesics and monitored until the attack subsided in order to determine the duration and severity. The level of incapacitation was assessed by the response to the question as to whether the affected were completely or partly unable to work and the duration for which they were incapacitated.

\subsubsection{Statistical analysis}

All data were entered in a database program Dbase IV and analysed using the SPSS (Statistical package for Social Scientists version 4). Univariate analysis using $\chi^{2}$ tests were done to assess the association between ADL and age, sex, chronic manifestations of lymphoedema, its grade and duration. For males the association between ADL with hydrocele and its size was also assessed. Multivariate analysis using multiple logistic regression was performed to adjust for confounding variables. Only variables which were significantly associated with ADL in the univariate analysis were included in the logistic regression model. To assess the importance of hydrocele pathology as a predictor of ADL separate multivariate analysis was performed for males alone.

\section{Results}

Based on the cross-sectional morbidity survey, a total population of 4576 individuals was examined. Of these $3.1 \%$ (141) were seen to have chronic filariasis. Table 1 shows the proportion of those affected in each village.

\subsection{Incidence of acute filarial attacks}

During the prospective surveillance 3000 individuals were visited on a fortnightly basis and 26 visits were made (Table 2). In the 12 months of follow up, 283 individuals experienced a total of 422 acute illness episodes which needed medical attention. Of the 422 illness episodes recorded, 98 were confirmed as acute adenolymphangitis based on the criteria and an additional 67 episodes were classified as suspected ADL episodes, while the remaining 257 episodes were acute illnesses which did not meet the ADL criterion. The acute ill- nesses were mainly due to malaria, arthritic pains and upper respiratory infections. Based on the confirmed episodes the annual incidence of ADL was 33 per 1000 population.

The 98 ADL episodes recorded were experienced by 65 individuals. The number of ADL episodes per person ranged from one to five with the majority $(60.4 \%)$, having had one episode only. All individuals diagnosed as having an ADL attack, were seen during a recall period of 14 days. Of the 98 ADL episodes, 51 (52.0\%) were seen during the acute phase and the rest were seen when the acute phase was over.

The mean duration of an ADL episode was 8.6 days (range $2-21$ days). In $72.5 \%$ of the episodes the affected individuals were completely unable to work. The mean duration of total incapacitation was 3.7 days (range $1-14$ days).

The incidence of ADL was almost three times higher among males compared to females (52.7 per 1000 population among males, and 18.7 per 1000 population among females). The difference in the incidence of ADL between males and females was statistically significant $\left(\chi^{2}=17.3, P<\right.$ 0.001). In addition, ADL incidence showed an increasing trend with advancing age up to the age of 59 years when it started to decrease. This increasing trend in the incidence of ADL with age is statistically significant $\left(\chi^{2}\right.$ trend $=79.3, P<$ 0.001).

Table 3 shows the anatomical distribution of the ADL episodes. Of the 98 ADL episodes $61.3 \%$ occurred in the lower limbs, while $35.7 \%$ occurred in the scrotum.

\subsection{Relationship of acute adenolymphangitis and lymphatic pathology}

Individuals with lymphoedema were more likely to have ADL attacks compared to the normal exposed population. Those with lymphoedema, 61.3\% developed ADL during the 12 months follow up period, while only $2.6 \%$ without lymphoedema (normal exposed population) had ADL episodes $\left(\chi^{2}=333.7, P<0.001\right)$. However, among the individuals with lymphoedema the duration and the grade of lymphoedema was not significantly associated with $\operatorname{ADL}\left(\chi^{2}=0.35, P>\right.$ 
Table 2

Age and sex distribution of ADL Incidence per 1000 population

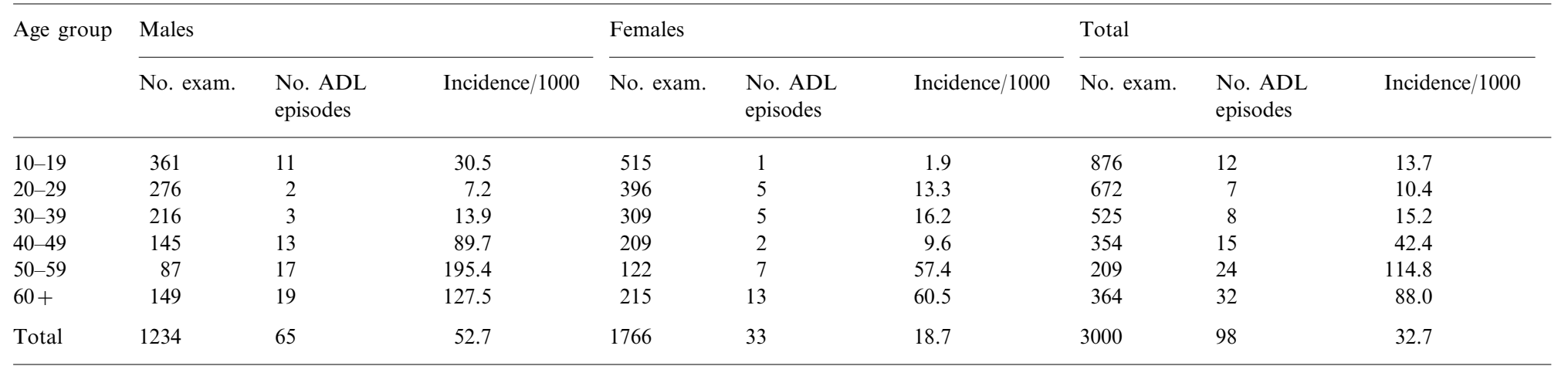


Table 3

Anatomical distribution of ADL episodes by sex

\begin{tabular}{|c|c|c|c|c|c|c|}
\hline \multirow[t]{2}{*}{ Anatomical site } & \multicolumn{2}{|l|}{ Males } & \multicolumn{2}{|l|}{ Females } & \multicolumn{2}{|l|}{ Total } \\
\hline & $\begin{array}{l}\text { No. ADL } \\
\text { episodes }\end{array}$ & $\begin{array}{l}\text { Proportion of the to- } \\
\text { tal }(\%)\end{array}$ & $\begin{array}{l}\text { No. ADL } \\
\text { episodes }\end{array}$ & Proportion & $\begin{array}{l}\text { No. ADL } \\
\text { episodes }\end{array}$ & Proportion $(\%)$ \\
\hline $\begin{array}{l}\text { Right lower } \\
\text { limb }\end{array}$ & 17 & 26.2 & 16 & 48.5 & 33 & 33.7 \\
\hline Left lower limb & 12 & 18.5 & 15 & 45.5 & 27 & 27.6 \\
\hline $\begin{array}{l}\text { Right upper } \\
\text { limb }\end{array}$ & & & 1 & 3.0 & 1 & 1.0 \\
\hline Left upper limb & 1 & & 1 & 3.0 & 2 & 2.0 \\
\hline Scrotum & 35 & 53.8 & & & 35 & 35.7 \\
\hline $\begin{array}{l}\text { Total ADL } \\
\text { episodes }\end{array}$ & 65 & 66.3 & 33 & 33.7 & 98 & $100 \%$ \\
\hline
\end{tabular}

0.05 and $\chi^{2}=1.87, P>0.05$ for duration and grade respectively).

Among males, the findings show increased incidence of ADL among those with hydrocele pathology and the association was found to be significant $\left(\chi^{2}=88.3, P<0.001\right)$.

Similar to the findings in the lymphoedema group, the size of hydrocele was not a significant factor in influencing the incidence of $\operatorname{ADL}\left(\chi^{2}=\right.$ $0.54, P>0.05)$. It was however noted that of all ADL cases in males, $80 \%$ of those with hydrocele pathology exhibited ADL of the scrotum as opposed to $20 \%$ with limb ADL. This suggests that hydrocele is a significant factor influencing the incidence of ADL of the scrotum rather than limb ADL.

Multiple logistic regression was done to investigate factors that can significantly predict ADL episodes. Findings show that although the incidence of ADL was found to be high among the males in bivariate analysis, when age and lymphoedema are controlled for, females are three times more likely to develop ADL than males (OR 3.78, (2.19-6.12)).

Further analysis shows that there is variation in individual age distribution with sex, with a tendency for females being younger than males (mean ages: 47.1 and 51.6 years, respectively). This suggests that the high ADL attacks among the males is due to either the high prevalence of chronic disease among them or due to the fact that males were on average older than the females. We tested for the interaction between sex and lymphoedema and between age and sex and in both cases there was no statistical significance. This suggests that the relationship between ADL and lymphoedema is similar for both sexes and that the increase in ADL incidence with age is also similar in both sexes.

When age and sex were controlled for it was found that individuals with lymphoedema were seven times more likely to develop ADL than the exposed normals $(\mathrm{OR}=7.33$, $(3.21-16.76)$ ).

To assess the influence of hydrocele in predicting ADL, a separate regression model was done for males alone. Results show that the effect of hydrocele in predicting ADL was not statististically significant $(\mathrm{OR}=0.7,(0.20-2.99))$. Similar to the findings in combined sexes model, age and lymphoedema were found to be significant predictors of ADL among the males $(\mathrm{OR}=5.96,(1.66-$ 21.4)) for lymphoedema and $(\mathrm{OR}=4.28$, (1.46-12.5)) for age.

\subsection{Seasonality and incidence of acute adenolymphangitis}

Analysis was carried out to delineate whether there was any seasonal variation in ADL occurrence. The results indicate an increasing trend in the incidence of ADL during the long rains (March to May), which peaked in May and again 
in August after the rains. However, there seem to be no significant difference in the proportion of ADL episodes between the rainy and the dry season (52.2 and 47.8\%, respectively (Fig. 1).

\section{Discussion}

'Filarial fevers' characterized by episodic attacks of adenolymphangitis, associated with fever and malaise, are believed to be an early sign of lymphatic filariasis (Manson-Bahr and Bell, 1987; WHO, 1992). Although this clinical syndrome is thought to be important in the development and progression of lymphatic filariasis (Pani et al. 1995) it has not been systematically characterized.

This study describes some of the epidemiological aspects of filarial adenolymphangitis in Tanzania where the annual incidence of ADL in Rufiji district was found to be 33 per 1000 population. This incidence rate is significantly high compared to earlier studies in other bancroftian filariasis endemic areas like India (Andhra Pradesh) where the incidence ranged between 8 and 27 per 1000 population/year (Rao et al., 1982a,b).

However, more recent studies in Ghana and India (Tamil Nadu) report significantly higher incidence rates of 96 and 97 per 1000 population per year, respectively, compared to this study (Gyapong et al., 1996; Ramaiah et al., 1996). This difference may be due to the variation in the constitution of the sample populations and differences in the prevalences of chronic disease manifestations in the different study sites.

Our results show that individuals with chronic manifestations of lymphoedema and hydrocele were at a higher risk to develop ADL compared to 'normal' exposed individuals. The significant association of chronic disease with ADL incidence has been reported in earlier studies both in Tanzania and from other endemic areas (Jordan, 1955; Pani et al., 1995; Gyapong et al., 1996; Ramaiah et al., 1996)

The increased frequency of ADL episodes in individuals with chronic manifestations and lymphoedema in particular is difficult to explain, since the aetiology of ADL is still not well characterized. The available information on ADL aetiology point to various factors; bacterial infections, toxins liberated from the adult parasites, immunological mechanisms and repeated exposure to infective mosquito bites have all been implicated (Chaterjee, 1965; Evert et al., 1980; Ottesen et al., 1982; Partono, 1987; Olszewski et al., 1993; Kar

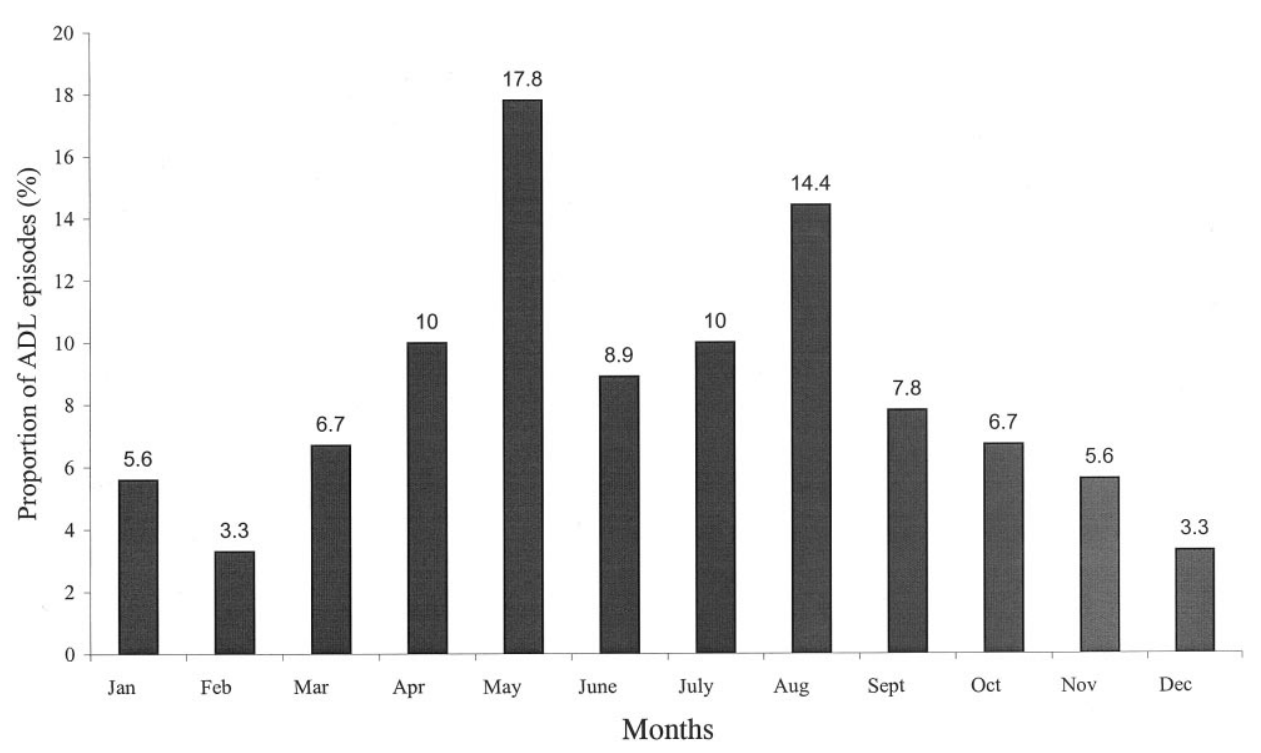

Fig. 1. Distribution of acute adenolymphangitis (ADL) episodes over a period of 12 months. 
et al., 1993; Addis et al., 1994; Olszewski et al., 1997). However, the aetiology of secondary bacterial infections may be a plausible explanation given the fact that the skin of individuals with limb elephantiasis may be compromised and the risk of infection possibly increased by the frequent exposure to a potentially contaminated environment. This hypothesis is supported by recent observations that simple foot hygiene measures lower the incidence of ADL in individuals with lymphoedema (Burri et al., 1996; Shenoy et al., 1998).

The incidence of ADL was significantly higher in males than females. This observation is similar to what is generally seen in other clinical manifestations of filariasis. Higher microfilaria rates and densities as well as higher prevalences of chronic manifestations have been reported in males than females (McMahon et al., 1981; Kimura et al., 1985).

Immunological as well as hormonal factors have been hypothesized as responsible for differential susceptibility (Eidinger and Ganet, 1972), however, the gender differences observed in ADL incidence in this study and other studies (Gyapong et al., 1996; Ramaiah et al., 1996), could be influenced by the differences in chronic disease prevalences and the differential susceptibility to ADL attacks for individuals with lymphoedema and hydrocele as seen in this study. The compounded pathology of hydrocele and lymphoedema that males manifest and overall advanced age in males that generally parallels increase in development of chronic manifestations, could explain the high incidence of ADL observed in males.

Unlike the observations of Pani et al. (1995) in India, where the frequency of ADL episodes were positively associated with the dynamic progression of lymphoedema through grade $1-3$, in this study there was no association with the grade of lymphoedema or the duration.

Similar observations of no association were found in Ghana (Gyapong et al., 1996). Based on the available data it is not clear whether there are underlying differences between the African and the Indian study populations, or simply a difference attributable to study designs. The African sites had a 2-week recall period compared to a 12-month recall period for the Indian site.

An increasing trend in ADL episodes with advancing age was observed and a high incidence of ADL episodes was found in the older age groups. This observation conforms to the fact that chronic manifestations of lymphatic filariasis also increases with age. However, it should be noted that in the younger age group of 10-19 years believed to have relatively low prevalences of chronic manifestations, a high ADL incidence of 14 per 1000 population was observed. It is possible that the total disease burden in this age group may have been underestimated and therefore, the effect of age in ADL attacks is difficult to explain.

The mean duration of an ADL attack in this study was found to be 8.6 days (range 2-21 days). This prolonged period, seems to exacerbate the morbidity and physical incapacitation associated with ADL attacks. It is notable that in $72.5 \%$ of the episodes the affected were totally incapacitated for a period of 4 days and unable to perform any work. Based on socio-economic studies on the same population (unpublished), it was observed that the acute attacks significantly reduce the number of hours the patient would have worked under normal circumstances. For example an average of $1.4 \mathrm{~h}$ of work were reported by individuals experiencing acute attacks compared to $5 \mathrm{~h}$ of work reported by controls, who experienced other illnesses during that period. Physical incapacitation does not only include failure to perform economic activities but may extend to loss of ability for personal care during the attacks.

The significant physical incapacitation associated with ADL attacks, shows that the disease burden due to filariasis is grossly increased by this condition. The effect of an ADL attack is not limited to the affected individual, but extends to other members of the family and community as the affected consistently need care and support in economic activity performance when they have the acute attack.

The incidence of ADL seemed to peak during the rainy season and 3 months after the rain. During this period the vector species of Culex quinquefasciatus and Anopheles gambiae are quite abundant. It is possible therefore that the peak 
incidence observed may be related to the increased transmission during this period when the mosquito populations and hence infective bites increase. This is consistent with the hypothesis that ADL attacks may be associated with an allergic response to massive parasite antigen release (Kar et al., 1993; Addis et al., 1994). However, the possible additional role of bacterial infections in ADL attacks, in a community where there may be increased environmental contamination during the rainy season cannot be ruled out.

The criterion used for the diagnosis of ADL seem to be quite specific, as it was possible to differentiate the other forms of acute illnesses which are quite rampant in the endemic communities. The symptoms/signs of lymphatic system involvement including, lymphadenitis, lymphangitis and cellulitis were significantly reported by individuals confirmed to have ADL. It is interesting to note that these signs were significantly low in other acute illnesses.

Although some of the symptoms/signs in the criterion used for diagnosis may be similar to those observed in bacterial lymphoadenopathy, the criterion was designed to exclude other likely conditions. Additional parasitological and or immunological tests would have complemented the criterion used for diagnosis. However, this study was designed to utilize a simple diagnostic tool for clinical diagnosis of ADL at community level and was not designed for detecting circulating filarial antigens or microfilaria in ADL cases. Reports by Addis et al. (1994), Pani et al. (1995), Gyapong et al. (1996), Ramaiah et al. (1996) suggest that the lack of these additional diagnostic tests is not likely to affect the reliability/validity of the results, since the study site has a long standing endemicity for lymphatic filariasis (Table 1).

In conclusion, this study clearly shows that ADL may be occuring more often than was hitherto believed and that the affected individuals are frequently incapacitated for long periods of time. This physical incapacitation interferes with personal care and negatively impacts on economic performance. This situation emphasizes the urgency and need to elucidate the aetiology of the acute attacks in endemic areas, with an aim to prevent filarial morbidity and debilitation in those already having chronic manifestations. Of immediate practicality is the promotion of the already developed simple hygienic measures for individuals with lymphoedema in order to minimize pain and frequency of morbidity.

\section{Acknowledgements}

We wish to thank the Rufiji community whose participation was central to the success of this work. The contribution and commitment of the research assistants and clinical officers ( $\mathrm{L}$. Mayembe, M. Kombo and J. Bulemera) is greatly appreciated. We thank Prof. Marcel Tunner of the Swiss Tropical Institute, Basel, for his coordinating role in this multi-centre study. We also wish to acknowledge and thank Prof. J.N. Minjas of Mubimbili University College of Health Sciences, Dar-es-Salaam for reviewing the manuscript. This study was financially supported by the UNDP/World Bank/WHO special programme for Research and Training in Tropical Diseases.

\section{References}

Addis, G.D., Eberhard, M.L., Lammie, J.P., 1994. Filarial adenolymphangitis without filarial infection. Lancet 343, 597.

Burri, H., Loutan, L., Kumaraswami, V., Vijayasekaran, V., 1996. Skin changes in chronic lymphatic filariasis. Trans. R. Soc. Trop. Med. Hyg. 90, 671-674.

Chaterjee, P., 1965. Filariasis: In: Basu, A.K. (Ed.), Tropical Surgery. Butterworth, London, pp. 51-103.

Eidinger, D., Ganet, J.J., 1972. Studies on the regulatory effects of the sex hormones on the antibody formulation and stem cell differentiation. J. Exp. Med. 47, 273.

Evans, D.B., Gelband, H., Vlassoff, C., 1993. Social and economic factors and the control of lymphatic filariasis. a review. Acta Tropical 53, 1-26.

Evert, A., Reitmeyer, J.C., Folse, D., 1980. Chronic infections of cats with Brugia malayi and Streptococcus. SE Asian J. Trop. Med Publ. Health 2, 32-39.

Gyapong, O.J., Gyapong, M., Adjei, S., 1996. The epidemiology of acute adenolymphangitis due to lymphatic filariasis in Northern Ghana. Am. J. Trop. Med. Hyg. 54, 591-595.

Jordan, P., 1955. Bancroftian filariasis: an assessment of its economic importance in Tanganyika. Trans. R. Soc. Trop. Med. Hyg. 49, 271-279. 
Kar, S.K., Maria, J., Kar, P.K., 1993. Humoral and immune response during filarial fever in Bancroftian filariasis. Trans. R. Soc. Trop. Med. Hyg. 87, 230-233.

Kimura, E., Penaia, L., Spears, G.F.S., 1985. Epidemiology of subperiodic bancroftian filariasis in Samoa 8 years after control by mass treatment with DEC. Bull. WHO 63 (5), 869-880.

McMahon, J.E., Magayuka, S.A., Kolstrup, N., Mosha, F.W., Bushrod, F.M., Abaru, D.E., 1981. Studies on transmission and prevalence of Bancroftian filariasis in four costar villages of Tanzania. Ann. Trop. Med. Parasitol. 75 (4), 415-431.

Manson-Bahr, P.E.C., Bell, D.R., 1987. Mansons Tropical Diseases, ELBS with Bailliere Tindall, 19th edition, pp 353.

Matola, Y.G., 1985. Prospects of human malaria and Bancroftian filariasis in the lower Rufiji basin, Tanzania. II. Bancroftian filariasis. Trop. Geog. Med. 37, 108-113.

Michael, E., Bundy, D.A., Grenfell, B.T., 1996. Re-assessing the global prevalence and distribution of lymphatic filariasis. Parasitology 112 (4), 409-428.

Olszewski, W.L., Jamal, S., Dworczynski, A., Swoboda, E., Pani, S., Manokaran, G., Kumaraswami, V., Bryla, P., 1993. Bacteriological studies of skin tissue fluid and lymph in filarial lymphedema. Proceedings of International Society of Lymphologists, Washington, Lymphology (special issue).

Olszewski, W.L., Jamal, S., Manokaran, G., Pani, S., Kumaraswami, V., Kubicka, U., Lukomska, B., Dworczynski, A., Swobod, E., Meisel-Mikolijczyk, F., 1997. Bacterialogic studies of skin, tissue fluid, lymph, and lymph nodes in patients with filarial lymphoedema. Am. J. Trop. Med. Hyg. 57 (1), 7-15.

Ottesen, E.A., Weller, P.F., Lunde, M.N., Hussains, R., 1982. Endemic filariasis on a Pacific island II Immunologic.aspects: immunoglobulin, complement and specific antifilarial IgG, IgM and $\operatorname{IgE}$ antibodies. Am. J. Trop. Med. Hyg. 31, 953-961.

Pani, S.P., Yuwaraj, J., Vanamail, P., Dhanda, V., Michael, E., Grenfell, B.J., Bundy, D.A.P., 1995. Episodic adenolymphangitis and lymphoedema in patients with bancroftian filariasis. Trans. R. Soc. Trop. Med. Hyg. 89, $72-74$.
Partono, F., 1984. Filariasis in Indonesia. Clinical manifestations and basic concepts of treatment and control. Trans. R. Soc. Trop. Med. Hyg. 78, 9-12.

Partono, F., 1987. The spectrum of disease in Lymphatic filariasis. Filariasis Ciba Foundation Symp. 127, 15-31.

Raghavan, N.G.S., 1969. Clinical manifestations and associated epidemiological factors of filariasis. J. Comm. Dis. 1, 75-102.

Ramaiah, K.D., Ramu, K., Kumar Vijay, K.N., Guyatt, H., 1996. Epidemiology of acute filarial episodes caused by Wucheria bancrofti infection in two rural villages in Tamil Nadu, South India. Trans. R. Soc. Trop. Med. Hyg. 90, 639-643.

Rao, C.K., Rama Prasad, V.V.N., Narasimham, M.V.V.L., Sundaram, R.M., Dutta, K.K., Krishna Rao, H., Venkatanarayana, M., 1982a. Prevalence and incidence rates of microfilaraemia and filarial disease in the East Godavani district of Andhra Pradesh. Ind. J. Med. Res. 75, 23-27.

Rao, C.K., Rarna Prasad, V.V.N., Narasimham, M.V.V.L., Krishna Rao, H., Venkatanarayana, M., Krishna Rao, P., 1982b. Impact of control measures on the prevalence and incidence of bancroftian filariasis in East Godavani district of Andhra Pradesh. Ind. J. Med. Res. 75, 515-520.

Shenoy, R.K., Suma, T.K., Raj an, K., Kumaraswami, V., 1998. Prevention of acute adenolymphangitis in brugian filariasis: comparison of the efficacy of ivermectin and diethylcarbamazine, each combined with local treatment of the affected limb. Ann. Trop. Med. Parasitol. 92 (5), 587-594.

World Bank, 1993. World Development Report. Investing in Health. Oxford University Press, New York, pp. 1-329.

World Health Organization, 1992. Lymphatic filariasis: the disease and its control. Fifth report of WHO expert committee on Lymphatic Filariasis. Geneva, WHO Technical Report Series no. 821.

World Health Organization, 1993. WHO expert consultation at the protocol development workshop on social and economic impact of lymphatic filariasis. Madras, India.

World Health Organization, 1995. Tropical Diseases Research Progress 1975-94. Highlights, 1993-94. 12th Programme Report UNDP/World Bank/WHO/TDR. 